\title{
MEASURE-THEORETIC CHARACTERIZATIONS OF HEREDITARILY-NORMAL SPACES
}

\author{
JOSEPH HERTZLINGER \\ Polytechnic University \\ (Received March 16, 1991 and in revised form March 30, 1992)
}

\begin{abstract}
In this paper we characterize hereditarily-normal spaces in terms of the measure-theoretic properties of the lattice of closed sets. We then generalize from that lattice to other lattices. We apply the results to extremallydisconnected spaces.
\end{abstract}

KEY WORDS AND PHRASES. Hereditarily-normal topological spaces, two-valued measures, lattice of closed sets, extremally-disconnected topological space.

1991 AMS SUBJECT CLASSIFICATION CODES. 54D15, 54G05.

\section{INTRODUCTION}

We can devise two measure-theoretic characterizations of hereditarily-normal spaces. First, a space is hereditarilynormal if and only if every pre-measure is a measure. Second, a space is hereditarily-normal if and only if the set of measures greater than a given measure form a chain. We can then generalize these results to other lattices (the lattices of open sets, zero sets, cozero sets, etc.). When we apply the results to the lattice of open sets we can derive a few conclusions about extremally-disconnected spaces.

We begin by giving a few definitions (we note that our lattice methods and terminology are consistent with standard usage, e.g., Camacho [1], Eid [4], Szeto [8]):

DEFINITION 1. We can define a partial order on the set of two-valued nontrivial measures on a space $X$ as follows: $\mu_{1} \leq \mu_{2}$ if and only if $\mu_{1}(F) \leq \mu_{2}(F)$ for all closed sets $F$. We define $\geq$ in a similar manner. (We note that here we are referring to finitely-additive two-valued measures on the algebra generated by the lattice of closed sets.)

DEFINITION 2. A two-valued pre-measure $\Pi$ on a space $X$ is a set function from the collection of closed sets to $\{0,1\}$ such that for any pair of closed sets $F_{1}$ and $F_{2}$ :

$$
\begin{gathered}
\Pi(\varnothing)=0, \\
\text { if } F_{1} \subseteq F_{2} \text { then } \Pi\left(F_{1}\right) \leq \Pi\left(F_{2}\right), \\
\text { if } \Pi\left(F_{1}\right)=1 \text { and } \Pi\left(F_{2}\right)=1 \text { then } \Pi\left(F_{1} \cap F_{2}\right)=1, \\
\text { if } F_{1} \cup F_{2}=X \text { then either } \Pi\left(F_{1}\right)=1 \text { or } \Pi\left(F_{2}\right)=1 .
\end{gathered}
$$

The last condition can be compared with the corresponding condition for measures: 


$$
\text { if } \Pi\left(F_{1} \cup F_{2}\right)=1 \text { then either } \Pi\left(F_{1}\right)=1 \text { or } \Pi\left(F_{2}\right)=1 \text {. }
$$

DEFINITION 3. A space $X$ has the pre-measure property if and only if every pre-measure on $X$ is a measure.

DEFINITION 4. A space $X$ is hereditarily normal if and only if every subset $Y$ of $X$ is normal.

We can replace this with the equivalent but more useful definition:

DEFINITION 4A. A space is hereditarily normal if and only if for every subset $Y$ of $X$ and every pair of closed sets $F_{1}$ and $F_{2}$ the condition $F_{1} \cap F_{2} \cap Y=\varnothing$ implies that there exist closed sets $F_{3}$ and $F_{4}$ such that $F_{3} \cap F_{1} \cap Y$ $=F_{4} \cap F_{2} \cap Y=\varnothing$ and $Y \subseteq F_{3} \cup F_{4}$.

DEFINITION 5. A regular measure $\mu$ is a measure on the algebra generated by the lattice of closed sets such that $\mu(M)$ is the supremum of the measures of all closed sets inside $M$, A regular measure is also a maximal measure (i.e., there is no measure strictly greater than it). For a two-valued measure the sets of measure one form a closed ultrafilter.

DEFINITION 6. A space $X$ has the measure-tree property if and only if for all two-valued measures $\mu_{,} \mu_{1}$, and $\mu_{2}$ on the algebra generated by the lattice of closed sets the conditions $\mu \leq \mu_{1}$ and $\mu \leq \mu_{2}$ imply that $\mu_{2} \leq \mu_{1}$ or $\mu_{1}$ $\leq \mu_{2}$. We note that the partial order $\leq$ is the same as that defined in Definition 1 .

The term "measure-tree property" requires explaining. In a normal space the set of measures less than a given measure (see Theorem 1) form a partially ordered set in which measures branch outward from the regular measure. If the space does not have the measure-tree property those measures also branch inward. If the space has the measuretree property the measures form a tree in which the branches do not reunite. This is equivalent to saying that all measures greater than a given measure form a chain.

We also note that this property closely resembles an axiom suggested by the noted mathematician Lewis Carrol [2] (Sylvie and Bruno, Chapter 18, p. 425): Things greater than the same are greater than one another.

\section{MAIN RESULTS}

The following (see Eid [4] and Frolik [5]) is a measure-theoretic characterization of normal spaces:

THEOREM 2.1. A space $X$ is normal if and only if for every measure $\mu$ and every pair of regular measures $\mu_{1}$ and $\mu_{2}$, if $\mu_{1} \geq \mu$ and $\mu_{2} \geq \mu$ then $\mu_{1}=\mu_{2}$.

PROOF. Let us assume $X$ is normal. Let us consider measure $\mu$ and regular measures $\mu_{1}$ and $\mu_{2}$. Let $\mu_{1} \geq \mu$ and $\mu_{2} \geq \mu$. Let $\mu_{1} \neq \mu_{2}$ and let us derive a contradiction. If $\mu_{1} \neq \mu_{2}$ then there is a set $M$ belonging to the algebra generated by the topology such that $\mu_{1}(M)=1, \mu_{2}(M)=0, \mu_{1}\left(M^{\prime}\right)=0$, and $\mu_{1}\left(M^{\prime}\right)=1$. Since $\mu_{1}$ and $\mu_{2}$ are regular measures there exist closed sets $F_{1}$ and $F_{2}$ such that $\mu_{1}\left(F_{1}\right)=\mu_{2}\left(F_{2}\right)=1, \mu_{1}\left(F_{2}\right)=\mu_{2}\left(F_{1}\right)=0, F_{1} \subseteq M, F_{2} \subseteq M^{\prime}$, and $F_{1} \cap F_{2}=\varnothing$. We therefore have $\mu\left(F_{1}\right) \leq \mu_{2}\left(F_{1}\right)=0$ and $\mu\left(F_{2}\right) \leq \mu_{1}\left(F_{2}\right)=0$. Since $X$, is normal there exist open sets $G_{1}$ and $G_{2}$ such that $G_{1} \subseteq F_{1}, G_{2} \subseteq F_{2}$, and $G_{1} \cap G_{2}=\varnothing$. Let their complements be $F_{3}=G_{1}{ }^{\prime}$ and $F_{4}=G_{2}{ }^{\prime}$. Since $\mu_{1}\left(G_{1}\right) \geq \mu_{1}\left(F_{1}\right)=1$ and $\mu_{2}\left(G_{2}\right) \geq \mu_{2}\left(F_{2}\right)=1$ we have $\mu_{1}\left(F_{3}\right)=\mu_{2}\left(F_{4}\right)=0$. Therefore $\mu\left(F_{1}\right)=\mu\left(F_{2}\right)=0$. Let $F_{5}$ $=F_{1} \cup F_{3}$ and $F_{6}=F_{2} \cup F_{4}$. Since $\mu\left(F_{5}\right)=\mu\left(F_{1} \cup F_{3}\right)=0, \mu\left(F_{5}^{\prime}\right)=1$. Similarly $\mu\left(F_{6}{ }^{\prime}\right)=1$. Since $F_{3} \subseteq F_{5}$ and $F_{4}$ $\subseteq F_{6}$ we have $F_{5}{ }^{\prime} \subseteq F_{3}{ }^{\prime}$ and $F_{6}{ }^{\prime} \subseteq F_{4}{ }^{\prime}$. Since $F_{3}{ }^{\prime} \cap F_{4}{ }^{\prime}=\varnothing$ we have $F_{5}{ }^{\prime} \cap F_{6}{ }^{\prime}=\varnothing$. This means that $\mu\left(F_{5}{ }^{\prime} \cup F_{6}{ }^{\prime}\right)$ $=\mu\left(F_{5}^{\prime}\right)+\mu\left(F_{6}^{\prime}\right)=2$, which is impossible. The necessary contradiction has been achieved.

For the other direction let $X$ be not normal and let us construct a measure $\mu$ and regular measures $\mu_{1}$ and $\mu_{2}$ such that $\mu_{1} \neq \mu_{2}$ but $\mu_{1} \geq \mu$ and $\mu_{2} \geq \mu$. Since $X$ is not normal there are two disjoint closed sets $F_{1}$ and $F_{2}$ which cannot be separated by open sets. The collection of sets $\left\{G\right.$ is open $\mid F_{1} \subseteq G$ or $\left.F_{2} \subseteq G\right\}$ therefore has the finite intersection property. The open filter that it generates can be extended to an open ultrafilter which induces a measure $\mu$ such that $\mu(G)=1$ for $G$ in the collection. Let $F_{0}$ be an arbitrary closed set with measure one; $\mu\left(F_{0}^{\prime}\right)=0$ which means $F_{0}{ }^{\prime}$ is not in the collection. This means that $F_{0}^{\prime} \nsubseteq F_{1}$ and $F_{0}^{\prime} \nsubseteq F_{2}$ or - in other words $-F_{1} \cap F_{0} \neq \varnothing$ and $F_{2} \cap F_{0} \neq$ $\varnothing$. This means that the collection of sets $\{F$ is closed $\mu(F)=1\} \cup\left\{F_{1}\right\}$ has the finite intersection property. The filter that it generates can be extended to a closed ultrafilter. The regular measure based on it will be called $\mu_{1} ; \mu \leq \mu_{1}$ and $\mu_{1}\left(F_{1}\right)=1$. Using similar reasoning we can see there is a regular measure $\mu_{2}$ such that $\mu \leq \mu_{2}$ and $\mu_{2}\left(F_{2}\right)=1$. Since $F_{1} \cap F_{2}=\varnothing, \mu_{1} \neq \mu_{2}$ which means the required measures have been constructed. 
THEOREM 2.2. If a space $X$ has the pre-measure property then it has the measure-tree property. PROOF. Let $X$ have the pre-measure property and let $\mu, \mu_{1}$, and $\mu_{2}$ be measures on $X$ such that:

$$
\mu \leq \mu_{1} \text { and } \mu \leq \mu_{2} \text {. }
$$

Let $\Pi=\min \left(\mu_{1}, \mu_{2}\right)$. It is easy to see that $\mu \leq \Pi$.

The function $\Pi$ is also a pre-measure. Conditions (1.1)-(1.3) in Definition 2 are obviously fulfilled. To prove condition (1.4), we let $F_{1} \cup F_{2}=X$. Because $\mu(X)=1$ we also have $\mu\left(F_{1} \cup F_{2}\right)=1$. The function $\mu$ is also a measure and therefore $\mu\left(F_{1}\right)=1$ or $\mu\left(F_{2}\right)=1$. Since $\mu \leq \Pi, \Pi\left(F_{1}\right)=1$ or $\Pi\left(F_{2}\right)=1$. Therefore condition (1.4) is fulfilled. The function $\Pi$ is thus a pre-measure. Since $X$ has the pre-measure property, $\Pi$ is a measure. This is possible only if either

$$
\mu_{2} \leq \mu_{1} \text { or } \mu_{1} \leq \mu_{2}
$$

(If this weren't the case then there would be closed sets $F_{1}$ and $F_{2}$ such that $\mu_{1}\left(F_{1}\right)=\mu_{2}\left(F_{2}\right)=1$ and $\mu_{1}\left(F_{2}\right)=\mu_{2}\left(F_{1}\right)$ $=0$. This means that $\mu_{1}\left(F_{1} \cup F_{2}\right)=\mu_{2}\left(F_{1} \cup F_{2}\right)=1$. Since $\Pi=\min \left(\mu_{1}, \mu_{2}\right)$, we have $\Pi\left(F_{1} \cup F_{2}\right)=1$ but $\Pi\left(F_{1}\right)=$ $\Pi\left(F_{2}\right)=0$. This is incompatible with $\Pi$ being a measure.)

We have been able to derive (2.2) from (2.1). Therefore $X$ has the measure-tree property.

THEOREM 2.3. If space $X$ is hereditarily normal then it has the pre-measure property.

PROOF. Let $\Pi$ be a pre-measure on $X$. In order to show that it is a measure we let $F_{1}$ and $F_{2}$ be closed sets such that $\Pi\left(F_{1} \cup F_{2}\right)=1$. Let $Y=X \backslash\left(F_{1} \cap F_{2}\right)$. This means that $F_{1} \cap F_{2} \cap Y=\varnothing$. Since $X$ is hereditarily normal this implies that there exist closed sets $F_{3}$ and $F_{4}$ such that $F_{3} \cap F_{1} \cap Y=F_{4} \cap F_{2} \cap Y=\varnothing$ and $Y \subseteq F_{3} \cup F_{4}$. Let $F_{5}$ and $F_{6}$ be closed sets such that $F_{5}=F_{3} \cup\left(F_{1} \cap F_{2}\right)$ and $F_{6}=F_{4} \cup\left(F_{1} \cap F_{2}\right) . F_{5} \cup F_{6}=X$ and since $\Pi$ is a pre-measure $\Pi\left(F_{5}\right)=1$ or $\Pi\left(F_{6}\right)=1$. Without loss of generality let $\Pi\left(F_{5}\right)=1$. Let $F_{7}=F_{5} \cap\left(F_{1} \cup F_{2}\right)$.

Let us now prove $F_{2}=F_{7}$. We can do this point by point.

First, let point $x \in F_{2}$. Either $x \in F_{1}$ or $x \in F_{1}^{\prime}$. If $x \in F_{1}$ then $x \in F_{1} \cap F_{2}$ which means $x \in F_{5}$ and thus $x \in$ $F_{7}$. If, on the other hand, $x \in F_{1}^{\prime}$ then $x \in F_{2} \backslash F_{1}$. Since $F_{4}$ is disjoint from $F_{2} \backslash F_{1}, x \in F_{4}^{\prime}$. Since $x \in Y$ and $F_{3} \cup$ $F_{4}=Y, x \in F_{3}$. Therefore $x \in F_{5}$ and thus $x \in F_{7}$.

Second, let $x \in F_{7} . F_{7}=\left(F_{5} \cap F_{2}\right) \cup\left(F_{5} \cap F_{1}\right)$. Either $x \in F_{5} \cap F_{2}$ or $x \in F_{5} \cap F_{1}$. If $x \in F_{5} \cap F_{2}$ then $x \in$ $F_{2}$. On the other hand if $x \in F_{5} \cap F_{1}$ then $x \in F_{5}$. If $x \in F_{5}$ then either $x \in\left(F_{1} \cap F_{2}\right)$ or $x \in F_{1} \cap F_{2}$ or $x \in F_{3}$. If $x \in F_{1} \cap F_{2}$ then $x \in F_{2}$. Now examine $x \in F_{3}$. If $x \in F_{3}$ then, since $F_{3}$ is disjoint from $F_{1} \cap Y, x \in\left(F_{1} \cap Y\right)^{\prime}$. Since $F_{3} \subseteq Y, x \in Y$. Therefore $x \in F_{1}{ }^{\prime}$. On the other hand, since $x \in F_{7}, x \in F_{1} \cup F_{2}$. This means that $x \in F_{2}$.

We have thus shown that $F_{2}=F_{7}$ and therefore $F_{2}=F_{5} \cap F_{1} \cup F_{2}$. Since $\Pi\left(F_{5}\right)=1$ and $\Pi\left(F_{1} \cup F_{2}\right)=1$ therefore $\Pi\left(F_{2}\right)=1$.

Similarly if $\Pi\left(F_{6}\right)=1$ then $\Pi\left(F_{1}\right)=1$. In other words, either $\Pi\left(F_{1}\right)=1$ or $\Pi\left(F_{2}\right)=1$.

We have shown that if $\Pi\left(F_{1} \cup F_{2}\right)=1$ then either $\Pi\left(F_{1}\right)=1$ or $\Pi\left(F_{2}\right)=1$. Therefore $\Pi$ is a measure. This applies to all pre-measures which means that $X$ has the pre-measure property.

LEMMA 2.1. If $\mu$ is a measure on space $X, Y$ is a subspace of $X$, and $Z$ is a set in the algebra generated by the topology such that $Z \subseteq Y$ and $\mu(Z)=1$ then the set function $\mu_{Y}$ defined as $\mu_{Y}(F \cap Y)=\mu(F)$ for all closed sets $F$ is well-defined and $\mu_{Y}$ is a measure on $X$.

PROOF. Let $F$ be a closed set in $Y$. Let $\mu_{Y}(F)$ be evaluated in two different ways:

$$
\begin{aligned}
& \mu_{Y}(F)=\mu\left(F_{1}\right) \text { where } F_{1} \text { is closed and } F_{1} \cap Y=F, \\
& \mu_{Y}(F)=\mu\left(F_{2}\right) \text { where } F_{2} \text { is closed and } F_{1} \cap Y=F .
\end{aligned}
$$


Since $Z \subseteq Y$ and $F_{1} \cap Y=F=F_{2} \cap Y$ we obtain $F_{1} \cap Z=F_{2} \cap Z$. Since $\mu(Z)=1$ we also obtain $\mu\left(F_{1} \cap Z\right)=$ $\mu\left(F_{1}\right)$ and $\mu\left(F_{2} \cap Z\right)=\mu\left(F_{2}\right)$. This means that $\mu\left(F_{1}\right)=\mu\left(F_{2}\right)$ so that $\mu_{Y}$ is the same no matter how it is evaluated.

Most of the properties which determine if a set function is a measure are inherited by $\mu_{Y}$. The remaining property is whether $\mu_{Y}(Y)=1$. Since $Y=X \cap Y, \mu_{Y}(Y)=\mu(X)=1$ which implies that $\mu_{Y}$ is a measure.

THEOREM 2.4. If a space $X$ has the measure-tree property then it is hereditarily normal.

PROOF. It is easiest to show this using the contrapositive.

Let $X$ be not hereditarily normal. There must exist a subset $Y$ of $X$ such that $Y$ is not normal. We must have a measure $\mu$ on $Y$ and a pair of regular measures $\mu_{1}$ and $\mu_{2}$ on $Y$ such that $\mu \leq \mu_{1}$ and $\mu \leq \mu_{2}$ but $\mu_{1} \neq \mu_{2}$. We must therefore have sets $M_{1}$ and $M_{2}$ in the algebra generated by the inherited topology on $Y$ such that:

$$
\mu_{1}\left(M_{1}\right)=\mu_{2}\left(M_{2}\right)=1 \text { and } \mu_{2}\left(M_{1}\right)=\mu_{1}\left(M_{2}\right)=0
$$

Since these are regular measure we must have closed sets $F_{1}$ and $F_{2}$ in $Y$ such that:

$$
\mu_{1}\left(F_{1}\right)=\mu_{2}\left(F_{2}\right)=1 \text { and } \mu_{2}\left(F_{1}\right)=\mu_{1}\left(F_{2}\right)=0
$$

Let $\mu_{3}, \mu_{4}$, and $\mu_{5}$ be two-valued functions of sets in the algebra generated by the topology on $X$ such that for all $M$ in the algebra:

$$
\mu_{3}(M)=\mu(M \cap Y), \mu_{4}(M)=\mu_{1}(M \cap Y), \text { and } \mu_{5}(M)=\mu_{6}(M \cap Y)
$$

These are obviously measures. They also satisfy the conditions $\mu_{3} \leq \mu_{4}$ and $\mu_{3} \leq \mu_{5}$. If $X$ had the measure-tree property then they would satisfy either $\mu_{4} \leq \mu_{5}$ or $\mu_{5} \leq \mu_{4}$. Since $F_{1}, F_{2} \subseteq Y$ it follows that $F_{1}=F_{3} \cap Y$ and $F_{2}=F_{4} \cap Y$ where $F_{3}$ and $F_{4}$ are closed. We therefore have the following

$$
\begin{aligned}
& \mu_{4}\left(F_{3}\right)=\mu_{1}\left(F_{3} \cap Y\right)=\mu_{1}\left(F_{1}\right)=1, \\
& \mu_{5}\left(F_{4}\right)=\mu_{2}\left(F_{4} \cap Y\right)=\mu_{2}\left(F_{2}\right)=1, \\
& \mu_{4}\left(F_{4}\right)=\mu_{1}\left(F_{4} \cap Y\right)=\mu_{1}\left(F_{2}\right)=0, \\
& \mu_{5}\left(F_{3}\right)=\mu_{2}\left(F_{3} \cap Y\right)=\mu_{2}\left(F_{1}\right)=0 .
\end{aligned}
$$

The conclusion $\mu_{4}\left(F_{3}\right) \leq \mu_{5}\left(F_{3}\right)$ is not true for $F_{3}$ and $\mu_{5}\left(F_{4}\right) \leq \mu_{4}\left(F_{4}\right)$ is not true for $F_{4}$. The space $X$ therefore does not have the measure-tree property.

We have thus proved the contrapositive and thus the original theorem that if $X$ has the measure-tree property then it is hereditarily normal.

THEOREM 2.5. (Summing up.) The following concepts are equivalent: a) hereditary normality; b) the measuretree property; and c) the pre-measure property.

The above reasoning can also be applied to other lattices than topologies. For example, lattices of zero sets are hereditarily normal. (This is easy to see. Let $Z_{f}$ and $Z_{g}$ be zero sets of functions $f$ and $g$, respectively. The functions remain continuous on the subspace. The function $(|f|-|g|) /(|f|+|g|)$ is also continuous because $(|f|+|g|)$ is nonzero if $Z_{f}$ and $Z_{g}$ are disjoint in the subspace. The cozero sets of the function's positive and negative portions produce the necessary cozero sets.) The theorems in this paper can be used to prove the well-known fact that premeasures on such lattices are measures. Similarly, Boolean algebras are obviously hereditarily normal; they also have 
the pre-measure property.

It is easy to see that the lattice of open sets is a normal lattice if and only if the space is extremally disconnected (i.e., in an extremally-disconnected space any two disjoint open sets can be separated by closed sets). It is also easy to see that if $\mu_{1}$ and $\mu_{2}$ are measures on a space then $\mu_{1}(G) \leq \mu_{2}(G)$ for all open sets $G$ if and only if $\mu_{1}(F) \geq \mu_{2}(F)$ for all closed sets $F$. If we put these facts together we can easily derive the following theorems:

THEOREM 2.6. A space $X$ is extremally disconnected if and only if for every measure $\mu$ and every pair of minimal measures (a measure is a minimal measure if and only if there is no nontrivial measure strictly less than it for every closed set) $\mu_{1}$ and $\mu_{2}$, if $\mu_{1} \leq \mu$ and $\mu_{2} \leq \mu$ then $\mu_{1}=\mu_{2}$.

THEOREM 2.7. A space $X$ is hereditarily extremally disconnected if and only if for all two-valued measures $\mu, \mu_{1}$, and $\mu_{2}$ on $X$ the conditions $\mu \geq \mu_{1}$ and $\mu \geq \mu_{2}$ imply that either $\mu_{2} \geq \mu_{1}$ or $\mu_{1} \geq \mu_{2}$.

These theorems can be used to prove:

THEOREM 2.8. A normal extremally-disconnected space is hereditarily normal if and only if it is hereditarily extremally disconnected.

PROOF. Let $X$ be normal and hereditarily extremally disconnected and let us prove it is hereditarily normal. Let $\mu_{1}, \mu_{2}$, and $\mu_{3}$ be three measures such that $\mu_{1} \leq \mu_{2}$ and $\mu_{1} \leq \mu_{3}$. Let $\mu_{4}$ be a regular measure greater than $\mu_{2}$. and let $\mu_{5}$ be a regular measure greater that $\mu_{3}$. Both $\mu_{4}$ and $\mu_{5}$ are greater than $\mu_{1}$ and therefore, since the space is normal, $\mu_{4}=\mu_{5}$. This means that $\mu_{2} \leq \mu_{4}$ and $\mu_{3} \leq \mu_{4}$. The space is hereditarily extremally disconnected and therefore $\mu_{2}$ and $\mu_{3}$ are comparable (i.e., either $\mu_{2} \leq \mu_{3}$ or $\mu_{3} \leq \mu_{2}$ ). We have shown that any two measures greater than the same measure are comparable. The space is therefore hereditarily normal.

We can use similar reasoning to show that if a space is extremally disconnected and hereditarily normal then it is hereditarily extremally disconnected.

If we examine the lattice of zero sets in a Tychonoff space, we can see that since it is hereditarily normal, if the lattice of cozero sets is normal (i.e., if the space is an $F$-space - see Gillman and Jerison [6], Chapter 14) then it is hereditarily normal. We can also easily prove the well-known results that a $z$-ideal in an $F$-space belongs to a chain from a minimal ideal to a maximal ideal.

The obvious conclusion - the property of being an $F$-space is hereditary - is false. Dow [3] constructed an $F$-space with an open subspace which is not an $F$-space. This can be reconciled with the fact that the lattice of cozero sets in an $F$-space is hereditarily normal. The members of the normal descendant lattice on the subspace consist of those cozero sets inherited from the original space. However, not every cozero set in the subspace has been inherited from the original space. The cozero sets of a subspace can include additional sets if there are more continuous functions on the subspace than on the original space.

At this point we have two questions: 1) Are there any spaces which are normal and extremally disconnected but which are not hereditarily extremally disconnected? 2) Are there any spaces which are hereditarily normal and extremally disconnected but which are not discrete?

The answer to the first question is yes. The Stone-Cech compactification of the integers $(\beta N$, Example 111 in Steen and Seebach [7]) is the Stone space of a complete algebra. It is therefore normal and extremally disconnected. The power set of the integers modulo the ideal of finite sets is incomplete so there its Stone space is not extremally disconnected even thought it is a subspace of $\beta \mathbf{N}$. This means that $\beta \mathbf{N}$ is not hereditarily extremally disconnected even though it is normal and extremally disconnected.

The answer to the second question is also yes. If we consider the single ultrafilter (Example 114 in Steen and Seebach [7]) we can see that this space must be extremally disconnected because if there are two disjoint open sets, at least one of them does not belong to the particular ultrafilter and it must therefore be closed. The two sets can therefore be separated by clopen sets. The space must also be hereditarily normal. It is countable and thus hereditarily Lindelof. A regular hereditarily-Lindelof space is hereditarily normal. 


\section{REFERENCES}

1. CAMACHO Jr., J. On maximal measures with respect to a lattice, Int. J. Math. Math. Sci. 14 (1991), 93.

2. CARROL, L. The Complete Lewis Carrol, Vintage Books, New York, 1976.

3. DOW, A. $\mathrm{CH}$ and open subspaces of $F$-spaces, Proc. Amer. Math. Soc., $\underline{89}$ (1983), 341.

4. EID G. On normal lattices and Wallman spaces, Int. J. Math. Math. Sci., 13 (1990), 31.

5. FROLIK, Z. Prime filters with the C.I.P., Comm. Math. Univ. Carolinae, 13 (1972), 553.

6. GILLMAN L. and JERISON M. Rings of Continuous Functions, Van Nostrand, New York, 1960.

7. STEEN L. and SEEBACH Jr, J. A. Counterexamples in Topology, Springer-Verlag, New York-Heidelberg-Berlin, 1978.

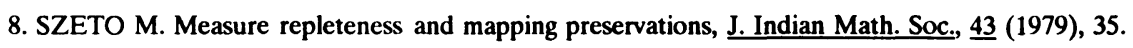




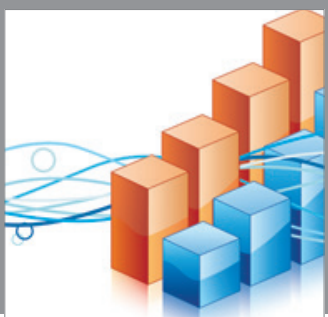

Advances in

Operations Research

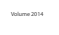

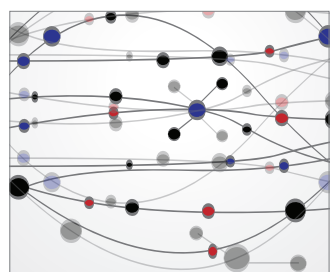

\section{The Scientific} World Journal
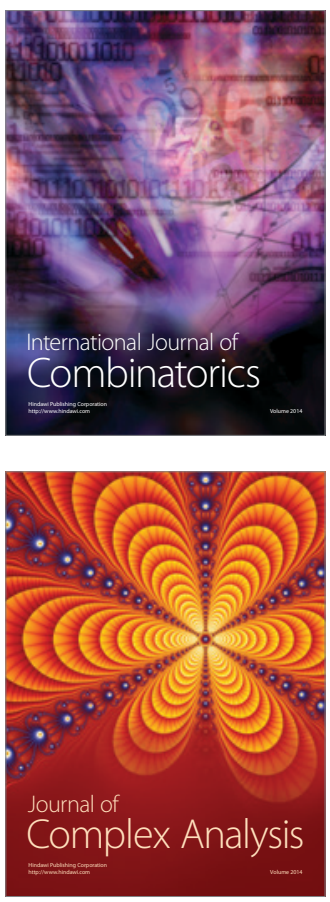

International Journal of

Mathematics and

Mathematical

Sciences
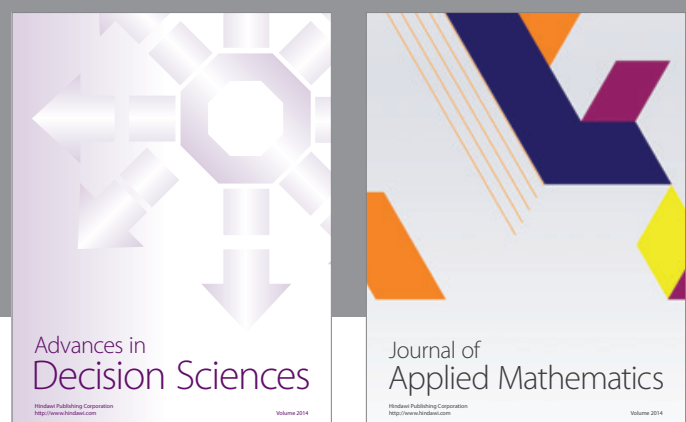

Journal of

Applied Mathematics
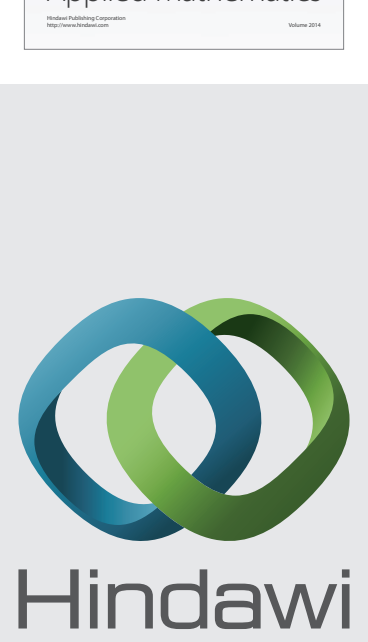

Submit your manuscripts at http://www.hindawi.com
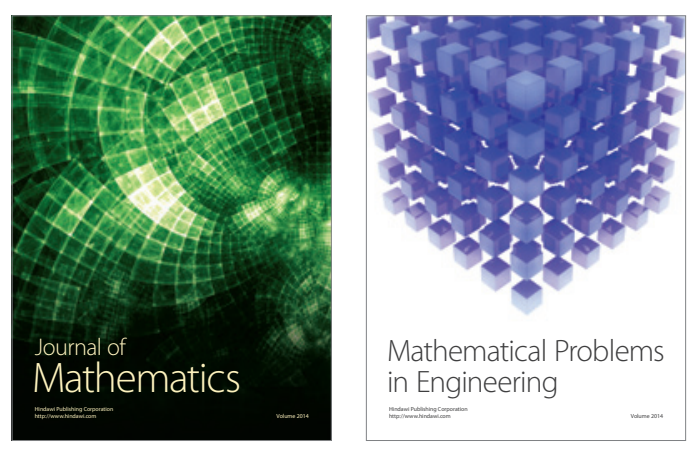

Mathematical Problems in Engineering
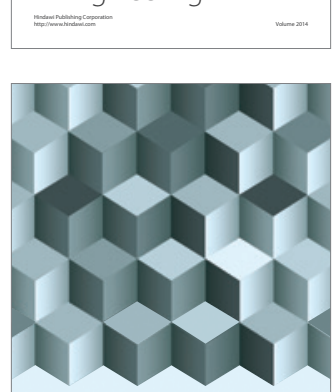

Journal of

Function Spaces
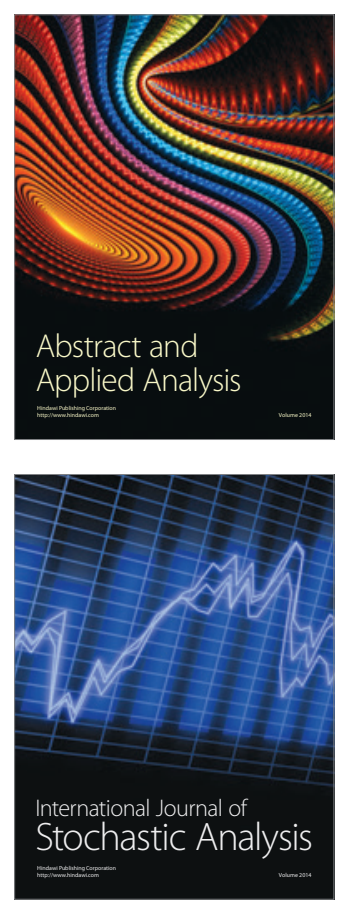

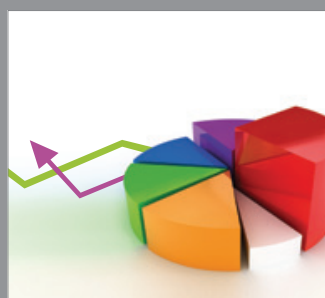

ournal of

Probability and Statistics

Promensencen
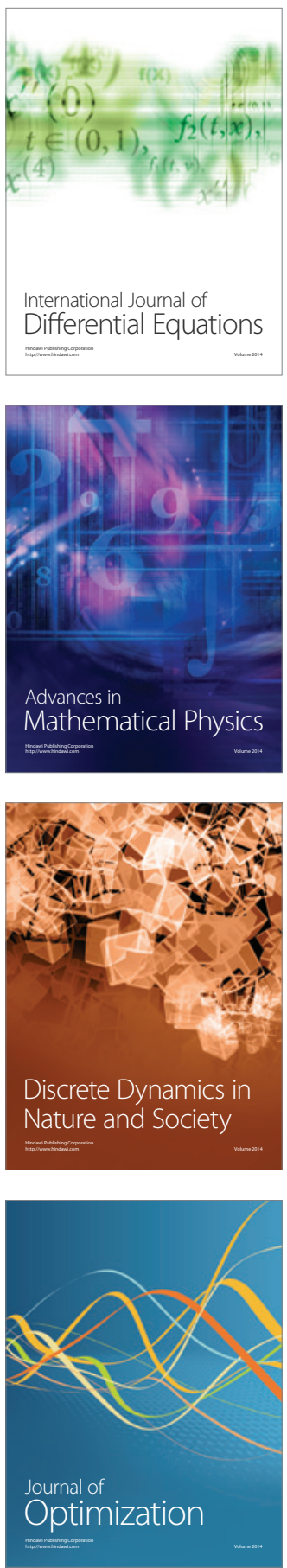